Ossifying fasciitis is a very rare disease of reactive character; however, it can mimic malignant lesions, especially osteosarcoma. We report a case of a 30-year-old woman, who experienced a rapidly growing painful lesion of the left knee joint, preceded by a trauma. The tumor was resected, and the histopathological image suggested a malignant lesion with features of an osteosarcoma. A detailed correlation with a clinicopathological and radiological analysis led to the final diagnosis of ossifying fasciitis at an extraordinary site of patellar retinaculum. Our case shows that the close similarity between ossifying fasciitis and osteosarcoma may be challenging.

Key words: fasciitis ossificans, ossifying fasciitis, osteosarcoma, knee, patellar retinaculum.

Contemp Oncol (Pozn) 2019; 23 (2): 121-125 DOI:https://doi.org/10.5114/wo.2019.85884

\section{Ossifying fasciitis at an extraordinary site - a case report and analysis of diagnostic pitfalls}

\author{
Łukasz Kowalczyk ${ }^{1}$, Marcin Braun ${ }^{1}$, Bartłomiej Tomasik², \\ Dominika Piasecka ${ }^{1}$, Dominika Michnoㄹ, Jacek Fijuth ${ }^{3}$, \\ Dorota Jesionek-Kupnicka ${ }^{1}$, Radzisław Kordek ${ }^{1}$
}

${ }^{1}$ Department of Pathology, Chair of Oncology, Medical University of Lodz, Poland ${ }^{2}$ Department of Biostatistics and Translational Medicine, Medical University of Lodz, Poland

${ }^{3}$ Department of Radiotherapy, Chair of Oncology, Medical University of Lodz, Poland

\section{Introduction}

Ossifying fasciitis is a disease classified as a variant of nodular fasciitis $[1,2]$. In general, nodular fasciitis is a relatively frequent pseudo-tumor of soft tissue; however its subtype ossifying fasciitis is extremely rare [3, 4]. Nodular fasciitis and ossifying fasciitis are detected mostly in adults, but the disease can also affect children - including infants [5, 6]. Nodular fasciitis is a benign non-neoplastic lesion of reactive character, localized in soft connective tissue [7]. Typically, ossifying fasciitis forms as a single heterotopic ossifying tumor within a few weeks [2]. Professional literature describes cases of fasciitis located mainly in the fascia or muscle of the upper extremities, chest, back, head and neck, and there are hardly any examples of localizations other than the musculoskeletal system [8]. Ossifying fasciitis typically has no tendency to recur or metastasize; even after a partial resection a regression may occur $[9,10]$. Histopathologically, nodular fasciitis and ossifying fasciitis can strongly mimic malignant lesions, mainly osteosarcomas. Thus, an accurate diagnosis of such lesions is critical and should include radiological and clinical data.

We present a case of a young woman with a very uncommon presentation of ossifying fasciitis of the patellar retinaculum, which strongly mimicked extraosseous osteosarcoma.

\section{Case report}

In January 2016, a 30-year-old woman was admitted to hospital because of pain in her left knee and limited knee joint mobility. The patient reported a traumatic event to her left lower limb within a period of six weeks.

Magnetic resonance imaging (MRI) of the left knee joint was performed in spin echo sequences (T1-weighted image, T2-weighted image) and inversion recovery sequences (turbo inversion recovery magnitude - TIRM), proton density (PD) fat saturated image and in T1-weighted image with the application of a contrast agent. A rupture of the medial patellar retinaculum with excessive lateral pressure syndrome was described. MRI also showed contrast-responsive tissue infiltration, sized $36 \mathrm{~mm} \times 16 \mathrm{~mm} \times 30 \mathrm{~mm}$, localized in the medial patellar retinaculum. A post-traumatic inflammatory lesion of the medial patellar retinaculum of the left knee was suggested (Fig. 1).

An arthroscopic medial retinacular repair with excision of the lesion was performed. On surgery, the tumor was well limited and separated from muscle and bone tissue.

In the macroscopic examination (Fig. 2), the surgical specimen was $5 \mathrm{~cm}$ $\times 4 \mathrm{~cm} \times 2 \mathrm{~cm}$ in size and it was of fibrous, fatty structure. It was well circumscribed, non-encapsulated, of higher density, with no signs of infiltration. 

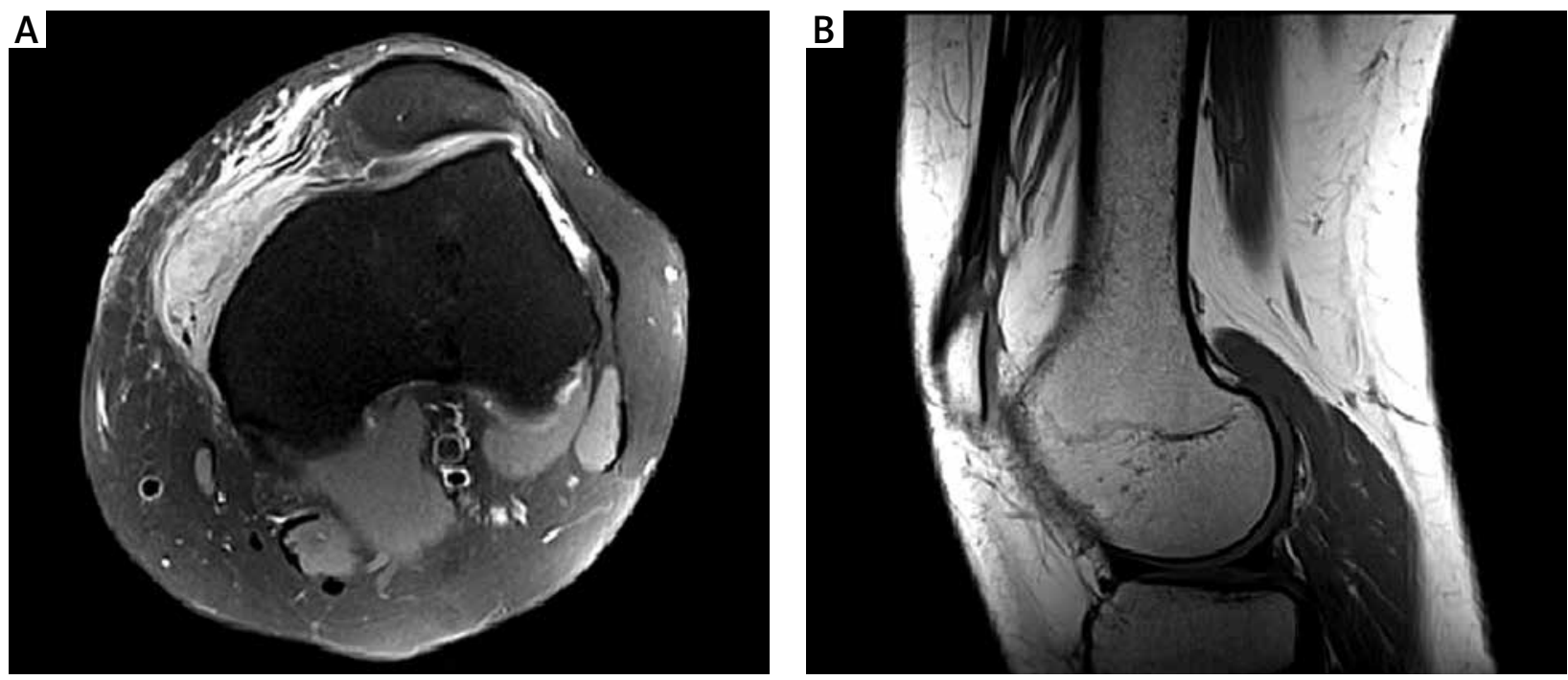

Fig. 1. Magnetic resonance imaging presentation of the tumor: The enhanced mass on axial view (proton density fat saturated image, turbo spin echo sequence) (A) and magnetic resonance imaging presentation of the tumor: Signs of dysplasia in the patellofemoral joint on sagittal view (T1-weighted image, turbo spin echo sequence) (B)
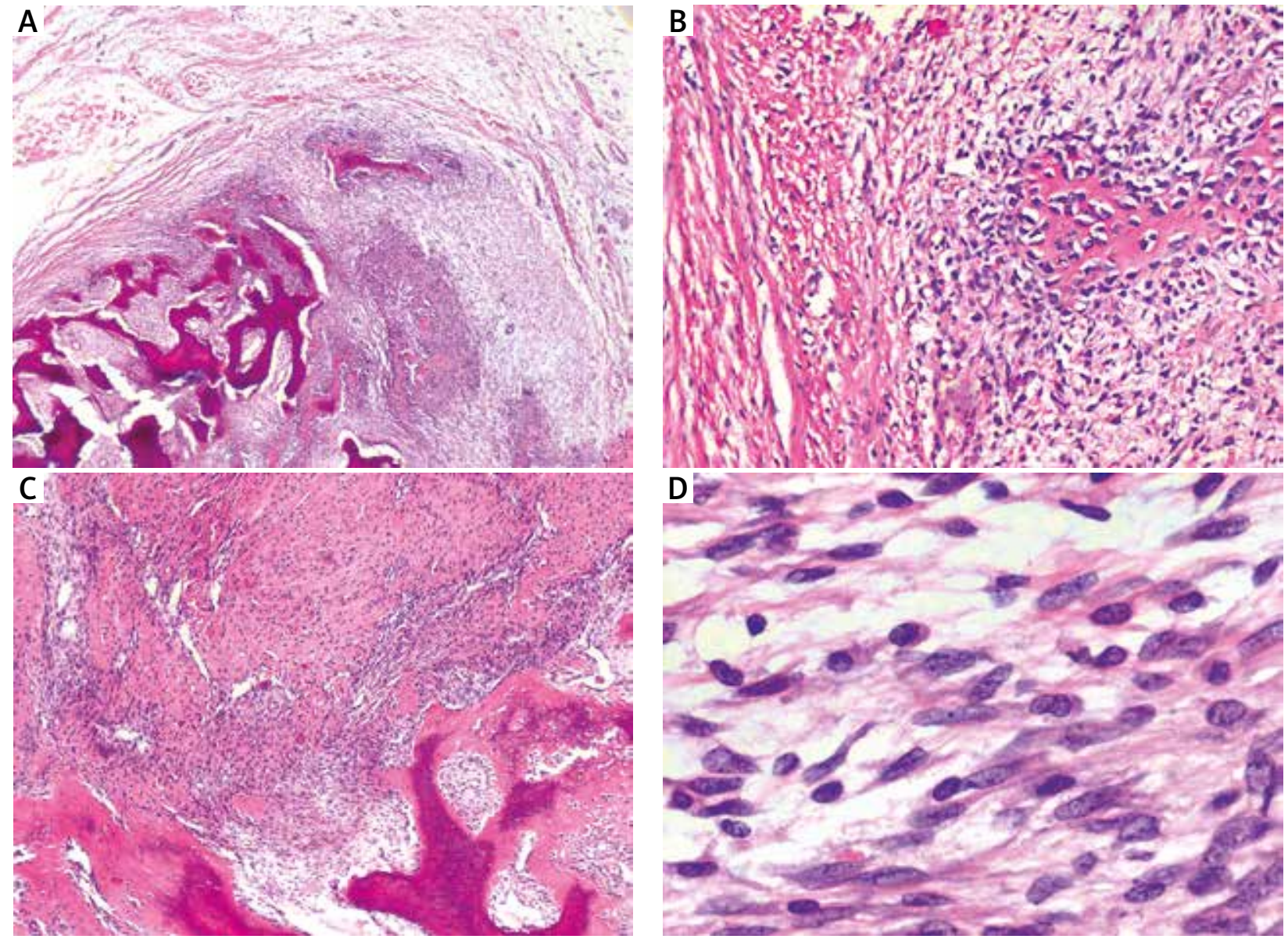

Fig. 2. The hematoxylin and eosin microscopic images show a non-capsulated, well-circumscribed tumor with heterogeneous structure - elements of fibrous connective tissue, hyalinization, cartilage, bone and osteoid formation visible. Magnification of 20× (A), 100× (B), $40 \times(C), 400 \times(D)$

Microscopically, the tumor was heterogeneous with extensive areas of intensive ossification. The tumor cells were grouped in "C" or "S" shaped bundles. There were holes and tears in the tumor structure as well as visible areas of carti- lage formation and hyalinization. Thin-walled blood vessels were scarce in the microscopic image. A weak inflammatory infiltration with scattered lymphocytes was seen. There were also areas with atypical cells and osteoid production. 

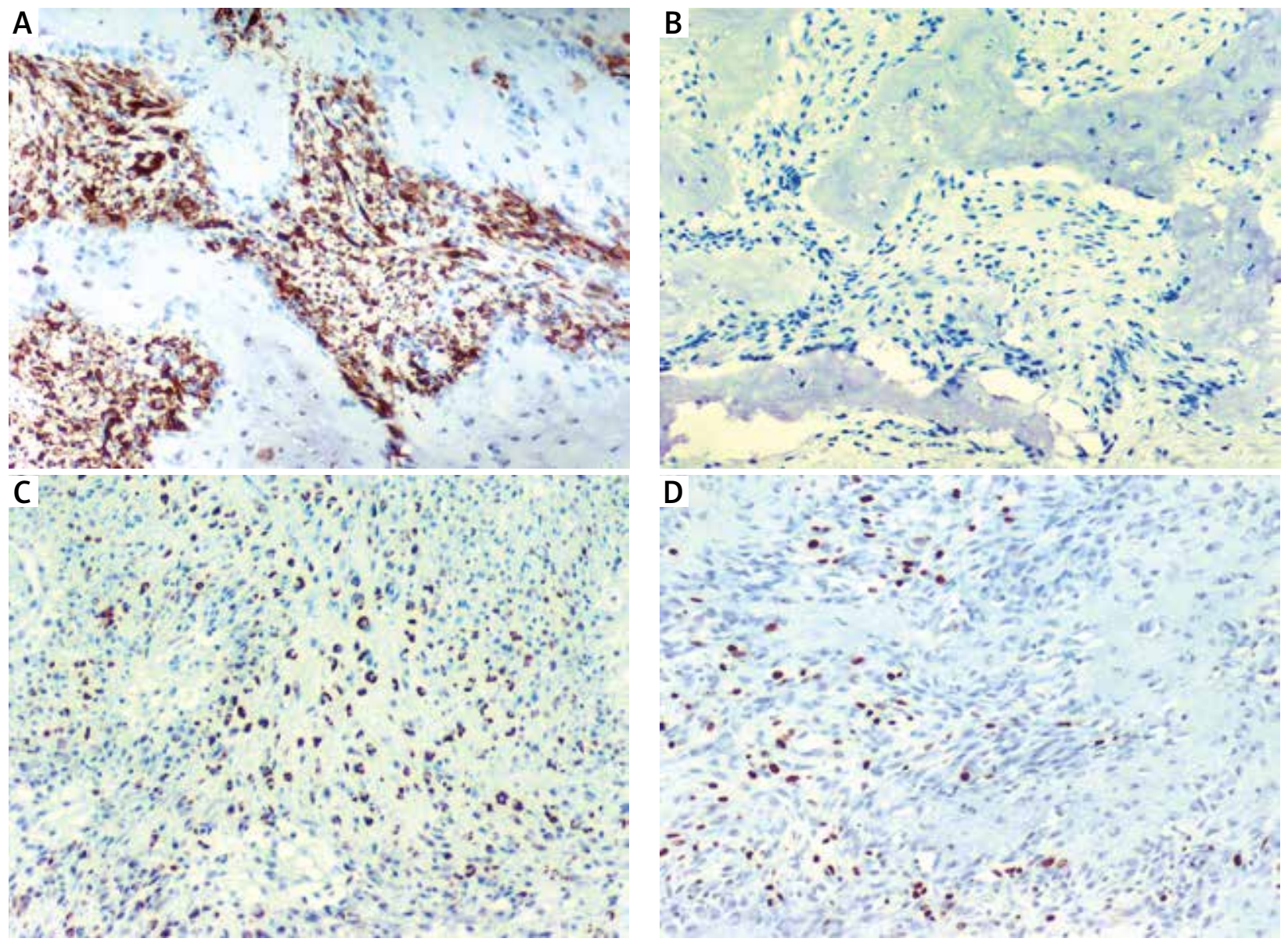

Fig. 3. Immunophenotype of the tumor: positivity for smooth muscle actin (A), negativity for S-100 protein (B), partial positivity for Myo-D1 (C), proliferation index of Ki-67 up to $5 \%$ (D). Magnification of $100 \times$

These fields resembled structures of an osteosarcoma. Mitotic figures were scarce (mitotic index of 1 per 20 high power fields). Foci of necrosis and giant cells were absent. An immunohistochemical analysis (Fig. 3) showed positivity for smooth muscle actin (SMA) and a weak partial positivity for MyoD1 and neuron-specific enolase (NSE). The lesion cells were negative for cytokeratins, desmin, S-100 protein, CD57, and CD246. The Ki-67 proliferation index was low, focally up to $5 \%$. A partial positive result for MyoD1 may suggest ossifying myositis, but the extra muscular localization of the tumor eliminates this possibility.

The obtained microscopic and radiological image as well as the location and clinical course (rapid development of a painful lesion) allow us to conclude that the detected disease is ossifying fasciitis.

The patient has remained under close surveillance for 18 months without signs of recurrence.

\section{Discussion}

We present a case of an extraordinarily located ossifying fasciitis nodule that strongly mimicked osteosarcoma and brought significant diagnostic difficulties.

Ossifying fasciitis, being a variant of nodular fasciitis, occurs as a superficially located tumor. Ossifying fasciitis is composed of fibrous connective tissue (richly cellular with uniform fibroblasts and immature myofibroblasts), cartilage, bone and osteoid [11]. Ossification in ossifying fasciitis is present focally and it consists of osteoid or mature lamellar bone trabeculae, in contrast to zonal pattern ossification, occurring in ossifying myositis [11]. In ossifying fasciitis, cells are diversiform and group in " $\mathrm{C}$ " or "S" shaped bundles. The image presents thin-walled blood vessels, extravasated erythrocytes and scattered leukocytes. Cells with pleomorphic features, various nuclei, evident nucleoli and atypical mitotic figures are rare. Chromatin is pale and regular. Ossifying fasciitis cells are stained positively with smooth muscle actin and vimentin, while they are negative for cytokeratins, desmin, myogenin, S-100 protein and anaplastic lymphoma kinase (ALK) [3]. The Ki-67 proliferation index is usually low [3].

Trauma-induced nodular fasciitis occurs only in 10-15\% of cases [3]. In the early phase of development of ossifying fasciitis (in its first week), hyperplasia of soft tissue takes place, similarly to nodular fasciitis. In the following weeks, bone tissue on various stages of differentiation becomes visible in the architecture [12]. The tumor reaches a characteristic macroscopic size $-3 \mathrm{~cm}$ on average - and induces pain and local inflammation [5]. It seems to be the primary symptom that initiates a diagnostic process. However, rare symptoms, such as peripheral neuropathy, were also described [13]. A radiological examination may 
reveal a soft-tissue lesion, characterized by various degree of swelling, calcification and contrast enhancement - depending on the phase of evolution $[7,14]$. The final diagnosis of a histopathological examination strictly involves clinical features. Ossifying fasciitis is usually treated by simple surgical excision. However, cases of ossifying fasciitis shrinkage after administration of anti-inflammatory medication have also been reported $[15,16]$. Metastases are extremely rare. The literature does not describe any case of progression of ossifying fasciitis towards malignant neoplasms.

Lesions which are morphologically similar to ossifying fasciitis may also occur in other diseases - both benign and malignant. An important diagnostic step concerns the exclusion of neoplastic character of the lesion. Differential diagnosis is challenging and tumors such as ossifying myositis or neuritis, fibromatosis, proliferative fasciitis or myositis, fibroma of the tendon sheath, myofibroma, myxoma, extraosseous osteosarcoma, and ossifying fibromyxoid tumor should be taken into consideration $[6,11]$.

One of the important discriminative attributes is the type of tissue from which the tumor comes, e.g. ossifying myositis derives from muscles, ossifying neuritis from nervous tissue, nodular fasciitis from connective tissue. Osteosarcoma seldom occurs in extraosseous locations (4-5\% of all osteosarcomas) and is most frequently located on extremities and the trunk (especially the thigh) $[17,18]$. It constitutes $1-2 \%$ off all sarcomas of soft tissue $[17,18]$. The majority of patients who suffer from extraosseous osteosarcoma are male and over 60 years old [17]. Unlike ossifying fasciitis, the course of extraosseous osteosarcoma is characterized by slow formation of a tumor. However, it may become larger than in ossifying fasciitis and is composed of pleomorphic cells $[3,19]$. In the microscopic image of sarcoma there are numerous atypical mitotic figures and chromatin is visibly coarse and irregular [3]. In extraosseous osteosarcoma we may spot focal necrosis, ossification and calcification accompanied by hemorrhage. Extraosseous osteosarcoma metastasizes commonly (mostly to bone and lung) and tends to recur locally. Extraosseous osteosarcoma is treated by surgical excision, chemotherapy and radiotherapy [20]. In our case, absence of high-grade atypia and scarce mitoses, the unusual location of the lesion, clear demarcation of the lesion and the history of trauma were grounds for exclusion of osteosarcoma.

Ossifying fibromyxoid tumor is an uncommon neoplasm of borderline malignant potential [21]. It typically occurs as a lesion of deep soft tissue that may be of a considerable size. Ossifying fibromyxoid tumor develops as well-circumscribed nodular mass, consisting of fibrous and myxoid portions [22]. Local recurrences and metastases during its course were described [22]. In our case, immunohistochemistry: NSE(-/+), Leu-7(-), S100(-), superficial location of the tumor, its size, lack of metastases and recurrence suggested a different diagnosis.

\section{Conclusions}

In conclusion, ossifying fasciitis is a pseudotumor which is similar to sarcoma in the clinical, radiologic and microscopic image. Active cooperation of an orthopedist, surgeon, radiologist, oncologist and pathologist significantly reduces the risk of misapplication of diagnostic tests as well as implementation of inadequate, aggressive and mutilating treatment.

The authors declare no conflict of interest.

\section{References}

1. Kwittken J, Branche M. Fasciitis ossificans. Am J Clin Pathol 1969; 51: 251-255.

2. Hutter RV, Stewart FW, Foote FWJ. Fasciitis. A report of 70 cases with follow-up proving the benignity of the lesion. Cancer 1962; 15: 992-1003.

3. Rosenberg AE. Pseudosarcomas of soft tissue. Arch Pathol Lab Med 2008; 132: 579-586.

4. Uhe S, Evert M, von Falkenhausen U, Freigang B. Fasciitis ossificans of the paranasal sinus. Ann Otol Rhinol Laryngol 2004; 113: 558-561.

5. Bernstein K, Lattes R. Nodular (pseudosarcomatous) fasciitis, a nonrecurrent lesion: clinicopathologic study of 134 cases. Cancer 1982; 49: 1668-1678.

6. Samaratunga H, Searle J, O'Loughlin B. Nodular fasciitis and related pseudosarcomatous lesions of soft tissues. Aust N Z J Surg 1996; 66: 22-25.

7. Leung LYJ, Shu SJ, Chan ACL, Chan MK, Chan CHS. Nodular fasciitis: MRI appearance and literature review. Skeletal Radiol 2002; 31: 9-13.

8. Hagino T, Ochiai S, Sato E, Watanabe Y, Senga S, Kondo T, Haro H. Intraarticular nodular fasciitis causing limitation of knee extension: A case report. Knee 2010; 17: 424-427.

9. Stanley MW, Skoog L, Tani EM, Horwitz CA. Nodular fasciitis: spontaneous resolution following diagnosis by fine-needle aspiration. Diagn Cytopathol 1993; 9: 322-324.

10. Kang A, Kumar JB, Thomas A, Bourke AG. A spontaneously resolving breast lesion: imaging and cytological findings of nodular fasciitis of the breast with FISH showing USP6 gene rearrangement. BMJ Case Rep 2015; pii: bcr2015213076.

11. Kempson RL, Fletcher CDM, Evans HL, Hendrickson MR, Sibley RK. Tumors of the Soft Tissues, Atlas of Tumor Pathology. Armed Forces Institute of Pathology, Washington 2001.

12. Lui PCW, Pang LM, Chu WCW, Tse GMK. Pathologic quiz case: a solitary breast nodule in an elderly woman. Fasciitis ossificans of the breast. Arch Pathol Lab Med 2004; 128: 29-30.

13. Rozen WM, Galloway S, Salinas C, Allen P, Schlicht S, Mann GB. Fasciitis ossificans with a radial neuropathy: a benign differential diagnosis for soft tissue sarcoma. J Clin Neurosci 2007; 14: 391-394.

14. Coyle J, White LM, Dickson B, Ferguson P, Wunder J, Naraghi A. MRI characteristics of nodular fasciitis of the musculoskeletal system. Skeletal Radiol 2013; 42: 975-982.

15. Risto O, Wahlström O, Abdiu A. The effect of low dose diclofenac sodium administered locally on heterotopic bone formation in rats. Int Orthop 1995; 19: 392-395.

16. Graham BS, Barrett TL, Goltz RW, Diego S. Nodular fasciitis: Response to intralesional corticosteroids. J Am Acad Dermatol 1999; 40: 490-492.

17. Hoch M, Ali S, Agrawal S, Wang C, Khurana JS. Extraskeletal Osteosarcoma: A case report and review of the literature. J Radiol Case Rep 2013; 7: 15-23.

18. Hishida T, Yoshida J, Nishimura M, Ishii G, Nakao M, Nagai K. Extraskeletal Osteosarcoma Arising in Anterior Mediastinum (case report). J Thorac Oncol 2009; 4: 927-929.

19. Bane BL, Evans HL, Ro JY, Carrasco CH, Grignon DJ, Benjamin RS, Ayala AG. Extraskeletal Osteosarcoma. Cancer 1990; 65: 27622770.

20. Goldstein-Jackson SY, Gosheger G, Delling G, et al. Extraskeletal osteosarcoma has a favourable prognosis when treated like con- 
ventional osteosarcoma. J Cancer Res Clin Oncol 2005; 131: 520 526.

21. Graham RP, Dry S, Li X, et al. Ossifying fibromyxoid tumor of soft parts: a clinicopathologic, proteomic, and genomic study. Am J Surg Pathol 2011; 35: 1615-1625.

22. Bakiratharajan D, Rekhi B. Ossifying fibromyxoid tumor: An update. Arch Pathol Lab Med 2016; 140: 371-375.

\section{Address for correspondence}

\section{Marcin Braun}

Department of Pathology

Chair of Oncology

Medical University of Lodz

251 Pomorska St.

92-213 Lodz, Poland

e-mail: braunmarcin@gmail.com

Submitted: 19.02 .2019

Accepted: 25.04 .2019 